\title{
What's New in Gastric and Esophageal Cancers
}

\author{
Kenneth Tanabe, $\mathrm{MD}^{1,2}$ \\ ${ }^{1}$ Division of Surgical Oncology, Massachusetts General Hospital, Boston, MA; ${ }^{2}$ Harvard Medical School, Boston, MA
}

The burdens imposed on society by gastric and esophageal cancer worldwide remain significant. But the light shines bright for new opportunities coming of age. A collection five outstanding articles are published together in this issue of Annals of Surgical Oncology to provide the reader with an understanding of the exciting, new directions of this field. These articles summarize a series of presentations made by their authors in a heavily attended session at the Society of Surgical Oncology Annual Cancer Symposia in Houston, Texas.

Mandeep Sawhney and Neil Sengupta provide insight into the latest success in endoluminal detection and treatment for early gastroesophageal cancers, ${ }^{1}$ including confocal laser microscopy, optical coherence tomography, thermal ablation techniques (i.e., radiofrequency, cryotherapy), and endoscopic submucosal dissection.

Controversy remains about whether gastroesophageal junction cancers should be treated as gastric cancers or as esophageal cancers. Based on existing data sets, perhaps most would agree that Siewert I tumors are appropriately classified and treated as esophageal cancers, and would similarly agree that Siewert III adenocarcinomas are best treated as gastric cancers.

Perhaps clinicians agree the least about Siewert II cancers. Interestingly, evidence-based treatment pathways for these cancers vary widely from each other. Nabil Rizk provides a rationale for treating Siewert II tumors from the approach of thoracic oncology, favoring neoadjuvant radiation therapy (particularly for tumors that extend into the intrathoracic esophagus), carboplatin and paclitaxel chemotherapy, and aggressive thoracic and abdominal lymphadenectomy. ${ }^{2}$

(C) Society of Surgical Oncology 2016

First Received: 17 April 2016;

Published Online: 8 September 2016

K. Tanabe, MD

e-mail: ktanabe@partners.org
John Mullen and colleagues present the counterpoint, citing the perspective of management for Siewert II tumors like gastric cancer is managed. ${ }^{3}$ They suggest that Siewert II cancers should be treated on the basis of the MAGIC trial (pre- or perioperative chemotherapy), with the addition of preoperative chemoradiation therapy for those with locoregionally advanced cancers and D2 lymphadenectomy. Jaffer Ajani and colleagues describe how somatic genotyping and other biomarkers currently are used to advantage in selecting treatments and developing new treatments, as well as recent results of clinical trials with immune checkpoint inhibitors. ${ }^{4}$ Vivian Strong and Gabriel Herrera describe where laparoscopic gastrectomy has been and, more importantly, where the field is moving. ${ }^{5}$

The field is evolving quickly, and the editors of Annals of Surgical Oncology are pleased to provide you with these updates in a single published volume.

\section{REFERENCES}

1. Sawhney S, Sengupta N. Advances in imaging, endoscopic and endoluminal therapies for early esophageal and gastric cancers. Ann Surg Oncol. 2016. doi:10.1245/s10434-016-5425-7.

2. Rizk N. Gastroesophageal junction tumors. Ann Surg Oncol. 2016. doi:10.1245/s10434-016-5427-5.

3. Mullen JT, Kwak E, Hong T. What's the best way to treat GE junction tumors? Approach like gastric cancer. Ann Surg Oncol. 2016. doi:10.1245/s10434-016-5426-6.

4. Elimova E, Song S, Shimodaira Y, Lin Q, Ajani, J. Exploiting molecular and immune biology of gastric and gastroesophageal adenocarcinomas to discover novel therapeutic targets. Ann Surg Oncol. 2016. doi:10.1245/s10434-016-5428-4.

5. Strong V. Minimally invasive gastroesophageal surgery. Ann Surg Oncol. 2016. doi:10.1245/s10434-016-5429-3. 